\title{
A Implantação do Princípio do Destino na Cobrança do ICMS e suas Implicações Dinâmicas sobre os Estados*
}

\author{
Nelson Leitão Paes ${ }^{\dagger}$
}

\author{
Contents: 1. Introdução; 2. O ICMS Interestadual; 3. Metodologia; 4. Discussão dos \\ Resultados; 5. Considerações Finais; A. Tabelas. \\ Keywords: ICMS; Princípio do Destino; Equilíbrio Geral; Finanças Públicas. \\ JEL Code: $\quad$ H71; C68; H2O.
}

Neste artigo, utilizou-se um modelo de equilíbrio geral dinâmico para estimar os efeitos econômicos e sobre a arrecadação dos Estados da adoção do princípio do destino na tributação do ICMS, de longe o principal tributo brasileiro. Os resultados mostram impactos importantes sobre a arrecadação dos Estados, com ganhos de quase $40 \%$ no Piauí, e perda de mais de 13\% no Espírito Santo, o maior prejudicado. De maneira geral, os Estados menos desenvolvidos são beneficiados pela nova sistemática, contribuindo para a redução das desigualdades na distribuição da arrecadação na federação, mas com pouco efeito sobre o produto e o consumo. In this paper, we used a dynamic general equilibrium model to estimate the effects of the adoption of destination principle at ICMS, the most important Brazilian tax, on the states public finance. The results had shown that Piauí could increase its revenue from ICMS by almost 40\%, while Espirito Santo could suffer a loss by more than $13 \%$. In general, less developed states will gain with the adoption of destination principle, contributing to reduce the inequality of tax revenues between states in Brazil, with little effect in output and consumption.

\section{INTRODUÇÃO}

A desigualdade regional é um dos traços mais marcantes e persistentes da federação brasileira. Superá-la consiste de um desafio de grandes proporções e envolve aspectos econômicos, sociais e culturais, entre outros. O presente trabalho busca enfocar como a forma de tributar o Imposto sobre Circulação de Mercadorias e Serviços (ICMS), principal tributo brasileiro, contribui atualmente para o aprofundamento das diferenças econômicas entre os Estados e como a alteração na sistemática da cobrança abre a possibilidade para o seu enfrentamento.

\footnotetext{
*O autor agradece aos comentários de um parecerista anônimo, assumindo inteira responsabilidade por qualquer erro remanescente.

†Programa de Pós-Graduação em Economia - PIMES/UFPE. End: Av. Professor Morais Rego, s/n, Centro de Ciências Sociais Aplicadas, Cidade Universitária, Recife-PE, CEP 50.670-901. E-mail: nlpaes@gmail .com.
} 
Apenas para contextualizar a situação presente, a arrecadação própria per capita de dois Estados nordestinos como Piauí e Maranhão corresponde apenas a $25 \%$ e $31 \%$, respectivamente, da média brasileira, situação atenuada, mas longe de ser resolvida, após as transferências verticais da União, quando a disponibilidade de recursos sobe para 54\% no Maranhão e $66 \%$ no Piauí ${ }^{1}$.

Em certa medida, a situação desfavorável em termos de disponibilidade de recursos estaduais per capita de alguns Estados decorre da adoção do princípio da origem na tributação do ICMS nas transações interestaduais. Por este princípio, parte substancial da arrecadação deste tributo se destina ao Estado produtor da mercadoria. Com isto, o ICMS perde o seu caráter de imposto sobre o consumo, tornando-se um tributo misto, incidindo tanto sobre a produção quanto o consumo.

A proposta que será aqui analisada examina os efeitos econômicos e sobre a arrecadação dos Estados, da adoção do princípio do destino, no qual o ICMS é repassado integralmente ao Estado onde a mercadoria é consumida, recolocando o imposto novamente na condição de um autêntico tributo sobre o consumo. Tal mudança promoverá uma razoável redistribuição de recursos com clara vantagem para os Estados consumidores em detrimento dos produtores.

Não é por outro motivo que a adoção do princípio do destino sempre se constituiu num dos principais óbices para a implantação da Reforma Tributária, uma vez que, os Estados produtores se colocam em posição contrária, temendo a perda de receitas. Adicionalmente, deve-se ressaltar o peso que o ICMS tem na arrecadação dos Estados, que na maioria deles, ultrapassa os $80 \%$.

Na literatura nacional são poucos os trabalhos que exploram o impacto quantitativo da mudança da sistemática da cobrança do ICMS. O primeiro a estimar a redistribuição de receitas decorrente da adoção do princípio do destino, ainda que superficialmente, foi o artigo de Varsano Varsano (1997), mas foi o trabalho de Paes and Siqueira (2005) que adotando um modelo de equilíbrio geral, calculou perdas e ganhos para os Estados. Tal artigo concentrou-se demasiadamente na demanda, adotando a hipótese de uma firma representativa para todo o país. O presente trabalho enriquece e aprofunda a análise ao incorporar uma firma e família representativa por Estado, alterando os resultados obtidos por aqueles autores.

A próxima seção trará uma rápida análise do ICMS e da forma de tributação das transações entre Estados. Na seção seguinte será apresentado o modelo de equilíbrio geral usado para simular a adoção do princípio do destino, sendo em seguida realizada a calibração. Na seção 4 os resultados serão discutidos e por último, a seção 5 traz os comentários finais.

\section{0 ICMS INTERESTADUAL}

Como se sabe o ICMS, um imposto estadual, é o principal tributo brasileiro, respondendo por aproximadamente $25 \%$ de toda a carga tributária nacional. Para bens produzidos ou importados e consumidos dentro do próprio Estado, todo o produto da arrecadação destina-se ao próprio ente federativo. Entretanto, nas transações interestaduais, a arrecadação do imposto se subdivide entre o Estado onde ocorreu a produção da mercadoria e o Estado onde ela é consumida. A alíquota padrão do ICMS é de $17 \%{ }^{2}$

Para o comércio interestadual valem as alíquotas definidas por resolução do Senado Federal. Os Estados do Norte (N), Nordeste (NE), Centro-Oeste (CO) e Espírito Santo (ES) possuem alíquotas mais benéficas do que o restante dos Estados do Sudeste (SE) e o Sul (S) como forma de incentivar o desenvolvimento dos primeiros. A regra para o comércio entre os Estados é resumida na tabela 1.

Assim, um produto vendido da Região Nordeste para a Região Norte terá alíquota total para o consumidor de $17 \%$, mas $12 \%$ pertencerão ao local de produção (NE) e $5 \%$ ao local de consumo (N). 0 mesmo resultado se aplica caso a venda fosse para um Estado do Sul ou Sudeste. Por outro lado, um

\footnotetext{
${ }^{1}$ Salvi (2005)

${ }^{2}$ É claro que nem todas as mercadorias são tributadas a 17\%, já que os Estados possuem competência para legislar a respeito do ICMS. Assim, encontram-se uma série de mercadorias com alíquotas superiores, tipicamente comunicações, combustíveis e bebidas, e outras com alíquotas menores, especialmente alimentos e medicamentos.
} 
Table 1: Distribuição das alíquotas do ICMS no comércio interestadual

\begin{tabular}{|l|c|c|}
\hline \multirow{2}{*}{\multicolumn{1}{|c|}{ Saída }} & \multicolumn{2}{|c|}{ Entrada } \\
\cline { 2 - 3 } N/NE/CO + ES & N/NE/CO + ES & S/SE (-ES) \\
S/SE (-ES) & $12 \%$ & $12 \%$ \\
& $7 \%$ & $12 \%$ \\
\hline
\end{tabular}

: Fonte: Elaboração do autor.

produto vendido da Região Sul para o Espírito Santo, da alíquota total de 17\%, 7\% pertence ao Estado produtor (S) e $10 \%$ ao consumidor (ES). A regra geral é que, saindo de uma região menos favorecida (N/NE/CO+ES), o produto paga sempre $12 \%$ na origem e $5 \%$ no destino. Quando ele sai de uma região mais favorecida (S/SE-ES), paga $7 \%$ na origem e $10 \%$ no consumo se for destinado ao $\mathrm{N} / \mathrm{NE} / \mathrm{CO}+\mathrm{ES}$, ou $12 \%$ na origem e $5 \%$ no consumo se for destinado ao S/SE-ES.

Portanto, atualmente, o Brasil aplica um princípio misto na tributação interestadual, com parte da arrecadação na origem e parte no destino.

Como uma fatia substancial dos recursos fica com o Estado produtor, torna-se interessante para os entes federativos expandir ao máximo a sua produção, aumentando a sua arrecadação, mesmo que nos limites do seu território não haja aumento do consumo. Melhor dizendo, o ICMS adquire em parte um caráter de imposto sobre a produção. ${ }^{3}$

Por outro lado, a adoção do princípio do destino implica que toda a arrecadação do ICMS passe para o Estado onde a mercadoria é consumida. Por esta razão há uma compreensível dificuldade a ser contornada e que ao longo dos últimos anos contribuiu para inviabilizar as propostas de reforma tributária. Espera-se que a adoção do princípio do destino represente perdas importantes de arrecadação nos Estados com maior produção e ganhos para os Estados consumidores. Dada a dificuldade de recursos pela qual todos os entes federativos vêm passando, não é surpresa que aqueles que têm a perder com a proposta se oponham a ela. Entretanto, tal restrição pode ser contornada com um período de transição razoável e o apoio federal durante esta passagem de forma a suavizar o custo de ajustamento daqueles que terão perdas.

A proposta que será aqui analisada consiste em se eliminar do sistema tributário brasileiro o princípio da origem para que seja adotado o princípio do destino. Na proposta a ser apresentada a eliminação do princípio da origem ocorrerá aos poucos.

Iniciando em 2004, as alíquotas interestaduais mostradas na tabela 1 seriam reduzidas em $50 \%$ em 2009 e totalmente eliminadas em 2014, quando finalmente toda a arrecadação do ICMS ficaria com o Estado de destino. O prazo de 10 anos é razoável e permite que os Estados prejudicados tenham uma transição mais suave com o apoio da União.

O Estado do Amazonas não será incluído no princípio do destino, pois é lá que fica localizada a Zona Franca de Manaus, local que conta com diversos incentivos fiscais, municipais, estaduais e federais. Pressupõe-se que eles serão mantidos, já que a sua eliminação reduziria em mais de $50 \%$ a arrecadação do Estado segundo nossos cálculos.

As simulações que serão realizadas são importantes para que se tenha uma idéia mais clara das conseqüências para cada ente federativo da nova sistemática de cobrança do ICMS. Por exemplo, Varsano (1997) estima que São Paulo perderia mais de 10\% da sua arrecadação total, enquanto Paes and Siqueira (2005) com uma metodologia com 27 famílias (uma por Estado) e apenas uma firma representativa nacional, encontra perdas importantes, entre 5\% e 15\% da receita, para São Paulo, Espírito Santo, Goiás, Mato Grosso do Sul e Santa Catarina. Entretanto, como se verá em seguida, ao se adotar um modelo

${ }^{3}$ Esta é uma das razões da existência da chamada "Guerra Fiscal". 
que contemple mais detalhadamente o lado da oferta, com a inclusão de 27 firmas representativas (uma para cada Estado), e torna mais realista o lado da demanda, ao permitir que a despesa pública estadual no único bem da economia seja também tributada pelo ICMS, as perdas tornam-se menos significativas.

\section{METODOLOGIA}

\subsection{Modelo}

A economia artificial está baseada no modelo neoclássico, é fechada, determinística e com população e tecnologia constantes. Há informação perfeita por parte de todos os agentes econômicos e os mercados são completos. Cada Estado possuirá uma firma e uma família representativa, diferenciada pela renda e pelo estoque de capital. Existe apenas um único bem nesta economia.

\subsection{Famílias}

Cada família fornece mão-de-obra e capital para a firma de seu Estado e cada firma contrata apenas capital e mão-de-obra da família do seu Estado. As famílias pagam impostos sobre consumo e renda, enquanto o governo transfere renda e demanda o único bem produzido por esta economia.

O modelo conta com 27 famílias representativas com vida infinita, cada uma com níveis de renda e consumo e estoque de capital inicial diferenciados. Para a diferenciação da renda das famílias, supõe-se que cada família possui uma produtividade por hora trabalhada fixa. Assim, cada hora de trabalho de cada família representará mais (ou menos) produção de acordo com esta produtividade.

As famílias resolvem um problema dinâmico, com preços e alíquotas tributárias dados e escolhem as seqüências de consumo, horas de trabalho e estoque de capital, no período seguinte, que maximizam a sua função utilidade (1).

$$
U_{i}=\sum_{i=1}^{\infty} \beta^{t}\left[\alpha \ln \left(c_{i t}\right)+(1-\alpha) \ln \left(1-h_{i t}\right)\right]
$$

em que $\beta$ denota o fator de desconto intertemporal, $\alpha$ o peso do consumo na função utilidade, $c_{i t}$ o consumo da família $i(i=1, \ldots, 27)$ no tempo $t$ e $h_{i t}$ as horas trabalhadas pela família $i$ no tempo $t$.

As famílias, entretanto, devem obedecer a restrição orçamentária (2) e ao fato de que o estoque de capital deve ser não negativo, ou seja, $k_{i t} \geq 0$.

$$
\begin{aligned}
\left(1+\tau_{F t}+s_{i} \sum_{j=1}^{27} a_{j i}\left(\tau_{E t}^{j i}+\tau_{I t}^{j i}\right)\right) c_{i t}-T_{i t}+k_{i t+1} & -(1-\delta) k_{i t} \\
& \leq\left(1-\tau_{h t}\right) \xi_{i} w_{t} h_{i t}+\left(1-\tau_{k t}\right) r_{t} k_{i t}
\end{aligned}
$$

Nestas expressões, $\tau_{F t}$ representa a alíquota do imposto federal sobre o consumo no tempo $t ; s_{i}$ representa a fração do consumo da família $i$ que é tributada pelo ICMS, já que principalmente serviços não sofrem a incidência deste imposto, $\tau_{E t}^{j i}$ a parte da alíquota do ICMS do consumo da família $i$ que é repassada para o Estado produtor $j ; \tau_{I t}^{j i}$ a parte da alíquota do ICMS do consumo da família $i$ de bens produzidos no Estado $j$ que fica com o Estado consumidor $i$ e $a_{j i}$ é a proporção no consumo da família $i$ de bens produzidos no Estado $j$. Observe que quando a família $i$ consome bens produzidos dentro do próprio Estado $\tau_{E t}^{j i}=0$. Por convenção, para estas variáveis, o primeiro índice ( $j$ no caso) corresponde ao local onde o bem foi produzido e o segundo índice ( $i$ no caso) é o local onde o bem foi consumido.

As demais variáveis e parâmetros são $\tau_{h t}$ a alíquota do imposto sobre a renda do trabalho pago pela família $i$ no tempo $t$; $w_{t}$ é o salário bruto da economia no tempo $t$; $\xi_{i}$ é a produtividade da família $i$; 
$T_{i t}$ a transferência governamental recebida pela família $i$ no tempo $t$ e $r_{t}$ é o preço antes dos impostos, do aluguel do capital no tempo $t, k_{i t}$ representa o estoque de capital da família $i$ no tempo $t$ e $\tau_{k t}$ é a alíquota do imposto sobre a renda do capital .

As respectivas condições de primeira ordem nos fornecem as seguintes equações:

$$
\begin{gathered}
h_{i t}=1-\frac{(1-\alpha)\left[1+\tau_{F t}+\sum_{j=1}^{27} a_{j i}\left(\tau_{E t}^{j i}+\tau_{I t}^{j i}\right)\right] c_{i t}}{\alpha\left(1-\tau_{h t}\right) \xi_{i} w_{t}} \\
c_{i t+1}=\frac{\left(1+\tau_{F t}+s_{i} \sum_{j=1}^{27} a_{j i}\left(\tau_{E t}^{j i}+\tau_{I t}^{j i}\right)\right)}{\left(1+\tau_{F t+1}+s_{i} \sum_{j=1}^{27} a_{j i}\left(\tau_{E t+1}^{j i}+\tau_{I t+1}^{j i}\right)\right)} \beta\left[(1-\delta)+\left(1-\tau_{k t+1}\right) r_{t+1}\right] c_{i t}
\end{gathered}
$$

\subsection{Firmas}

Já as 27 firmas são competitivas, contratando mão-de-obra $\left(h_{i t}\right)$ e capital $\left(k_{i t}\right)$ das famílias do seu estado. O problema da firma é basicamente estático, ou seja, ela escolhe as quantidades de insumos e produto que maximiza o seu lucro. A função de produção é do tipo Cobb-Douglas:

$$
y_{i t}=k_{i t}^{\theta}\left(\xi_{i} h_{i t}\right)^{1-\theta}
$$

onde $\theta$ é a participação da renda do capital na renda total e $y_{i t}$ é o produto estadual. Maximizando os lucros obtém-se a taxa de juros e o salário:

$$
\begin{gathered}
r_{t}=\theta k_{i t}^{\theta-1}\left(\xi_{i} h_{i t}\right)^{1-\theta} \\
w_{t}=\frac{w_{i t}}{\xi_{i}}=(1-\theta) k_{i t}^{\theta}\left(\xi_{i} h_{i t}\right)^{-\theta}
\end{gathered}
$$

\subsection{Governo}

São dois os tipos de governo. O governo estadual possui apenas um tributo, o ICMS, e a sua arrecadação decorre tanto das vendas realizadas dentro do Estado quanto das vendas de seus produtos realizadas em outros Estados. A restrição orçamentária dos governos estaduais é dada por:

$$
g_{i t}=\sum_{j=1}^{27} \tau_{I t}^{j i} a_{j i} s_{i}\left(c_{i t}+g_{i t}\right)+\sum_{j=1}^{27} \tau_{E t}^{i j} a_{i j} s_{j}\left(c_{j t}+g_{j t}\right)
$$

onde $g_{i t}$ é a despesa pública estadual com o único bem da economia. De forma a tornar o modelo mais próximo da realidade, as compras dos governos estaduais são também tributadas pelo ICMS. 0 governo federal arrecada impostos derivados de todos os demais tributos, à exceção do ICMS, para financiar seus gastos no único bem da economia e fazer transferências para as famílias.

$$
g_{F t}=\sum_{j=1}^{27} \eta_{i}\left(\tau_{F t} c_{i t}+\tau_{h t} \xi_{i} w_{t} h_{i t}+\tau_{k t} r_{t} k_{i t}\right)
$$

Em que $\eta_{i}$ é o percentual da população no Estado $i$ em relação a população total do Brasil. A restrição orçamentária federal é dada por: 


$$
\Gamma_{F t}=g_{F t}+T_{t}
$$

Em que $\Gamma_{F t}$ corresponde a arrecadação tributária no tempo $t, g_{F t}$ a despesa federal com o bem único da economia no tempo $t$ e $T_{t}$ é o total de transferências às famílias também no tempo $t$. O conjunto das seqüências relativas às variáveis fiscais $\left\{T_{i t}, g_{F t}\right\}$ e parâmetros tributários $\left\{\tau_{k t}, \tau_{F t}, \tau_{E t}^{j i}, \tau_{I t}^{j i}, \tau_{k t}\right\}$ é dado exógenamente, exceto a despesa estadual, calculada endogenamente por 8.

\subsection{Equilíbrio}

As famílias escolherão as seqüências $\left\{c_{i t}, h_{i t}, k_{i t}\right\}$ que maximizam sua utilidade sujeito a restrição orçamentária de cada uma, enquanto que as firmas representativas escolhem $\left\{h_{i t}, k_{i t}\right\}$ de forma a maximizar seus lucros, respeitando a restrição agregada da economia estadual:

$$
\sum_{j=1}^{27} a_{i j}\left(c_{j t}+g_{j t}\right)+k_{i t+1}-(1-\delta) k_{i t}+\gamma_{i} g_{F t}=k_{i t}^{\theta}\left(\xi_{i} h_{i t}\right)^{1-\theta}
$$

onde $\gamma_{i}$ é a fração da despesa federal no bem único produzido no Estado $i$. Para o cálculo do equilíbrio deve se resolver o sistema de equações de diferenças não-lineares composto por 3, 4, 6, 7, 8, 9 e 10, dado $k_{i 0}$.

A dinâmica do consumo das famílias é obtida substituindo 6 em 4:

$$
c_{i t+1}=\frac{\left(1+\tau_{F t}+s_{i} \sum_{j=1}^{27} a_{j i}\left(\tau_{E t}^{j i}+\tau_{I t}^{j i}\right)\right)}{\left(1+\tau_{F t+1}+s_{i} \sum_{j=1}^{27} a_{j i}\left(\tau_{E t+1}^{j i}+\tau_{I t+1}^{j i}\right)\right)}\left(\beta\left[(1-\delta)+\left(1-\tau_{k t+1}\right) \theta k_{i t+1}^{\theta-1}\left(\xi_{i} h_{i t+1}\right)^{1-\theta}\right] c_{i t}\right.
$$

Substituindo 7 em 3 tem-se a condição para as horas trabalhadas das famílias.

$$
h_{i t}=1-\frac{(1-\alpha)\left[1+\tau_{F t}+\sum_{j=1}^{27} a_{j i}\left(\tau_{E t}^{j i}+\tau_{I t}^{j i}\right)\right] c_{i t}}{\alpha\left(1-\tau_{h t}\right) \xi_{i}(1-\theta) k_{i t}^{\theta}\left(\xi_{i} h_{i t}\right)^{-\theta}}
$$

\subsection{Solução do Modelo}

Substituindo o consumo de 13 em 12 e em 11 e usando 8 para calcular $g_{i t}$, obtemos um sistema de equações composto por 8,11 e 12 em cada período $t$, e tendo como incógnitas as trajetórias de $\left\{h_{i t}\right\}_{t=0}^{T},\left\{k_{i t}\right\}_{t=0}^{T}$ e $\left\{g_{i t}\right\}_{t=0}^{T}$ para cada Estado. 


$$
\begin{aligned}
& g_{i t}=\sum_{j=1}^{27} \tau_{I t}^{j i} a_{j i} s_{i}\left\{\frac{\alpha\left(1-\tau_{h t}\right) \xi_{i}(1-\theta) k_{i t}^{\theta}\left(\xi_{i} h_{i t}\right)^{-\theta}\left(1-h_{i t}\right)}{(1-\alpha)\left[s_{i} \sum_{j=1}^{27} a_{j i}\left(\tau_{E t}^{j i}+\tau_{I t}^{j i}\right)\right]}+g_{i t}\right\}+ \\
& \sum_{j=1}^{27} \tau_{E t}^{i j} a_{i j} s_{j}\left\{\frac{\alpha\left(1-\tau_{h t}\right) \xi_{j}(1-\theta) k_{j t}^{\theta}\left(\xi_{j} h_{j t}\right)^{-\theta}\left(1-h_{j t}\right)}{(1-\alpha)\left[s_{j} \sum_{j=1}^{27} a_{i j}\left(\tau_{E t}^{i j}+\tau_{I t}^{i j}\right)\right]}+g_{j t}\right\} \\
& h_{i t+1}=1-\beta\left[(1-\delta)+\left(1-\tau_{k t+1}\right) \theta k_{i t+1}^{\theta-1}\left(\xi_{i} h_{i t+1}\right)^{1-\theta}\right] \frac{\left(1-\tau_{h t}\right) k_{i t}^{\theta} h_{i t}^{-\theta}\left(1-h_{i t}\right)}{\left(1-\tau_{h t+1}\right) k_{i t+1}^{\theta} h_{i t+1}^{-\theta}} \\
& k_{i t+1}=k_{i t}^{\theta}\left(\xi_{i} h_{i t}\right)^{1-\theta}-\sum_{j=1}^{27} a_{i j}\left\{\frac{\alpha\left(1-\tau_{h t}\right) \xi_{j}(1-\theta) k_{j t}^{\theta}\left(\xi_{j} h_{j t}\right)^{-\theta}\left(1-h_{j t}\right)}{(1-\alpha)\left[s_{j} \sum_{j=1}^{27} a_{i j}\left(\tau_{E t}^{i j}+\tau_{I t}^{i j}\right)\right]}+g_{j t}\right\} \\
& +(1-\delta) k_{i t}-\gamma_{i} g_{F t}
\end{aligned}
$$

Adotamos o período de transição até $T=50$. São, portanto, 1.377 variáveis de horas de trabalho (27 Estados x 51 períodos (0 a 50)), 1.377 variáveis para o gasto estadual e 1.323 variáveis do capital. A diferença ocorre porque o capital inicial de cada um dos 27 Estados da federação é dado, bem como pelo capital de estado estacionário, obtido por meio da equação 16 em estado estacionário:

$$
\frac{k_{i}}{h_{i}}=\tilde{k}_{s s}=\left[\frac{\frac{1}{\beta}-(1-\delta)}{\left(1-\tau_{k}\right) \theta \xi_{i}^{1-\theta}}\right]^{\frac{1}{1-\theta}}
$$

Como cada uma das equações 14, 15, 16 acima são aplicáveis para cada Estado, temos um total de $2 \times 27 \times 50$ (capital e horas de trabalho) $+27 \times 51$ (gasto estadual) $=4.077$ equações. Resolvemos este sistema não linear composto por 4.077 equações e 4.077 variáveis utilizando o método de Broyden para solução de sistemas não-lineares. ${ }^{4}$

\subsection{Calibragem}

3.7.1. Parâmetros Obtidos do Novo Sistema de Contas Nacionais do IBGE: 2000 - 2005

\subsubsection{Distribuição das Famílias}

A distribuição das famílias por Estado no modelo é dada pelo parâmetro $\eta_{i}$ com o uso dos dados das Contas Regionais 2004 do IBGE (2006) para determiná-lo.

${ }^{4}$ Para referência em relação ao método ver Miranda and Fackler (2002). 
Table 2: Parâmetros das Contas Nacionais

\begin{tabular}{|l|c|c|}
\hline Parâmetro & Descrição & Valor \\
\hline$\theta$ & Participação da Renda do Capital & 0,4751 \\
$\tau_{F}$ & Alíquota Trib. Consumo Federal & 0,1533 \\
$\tau_{h}$ & Alíquota Trib. Renda do Trabalho & 0,2202 \\
$\tau_{k}$ & Alíquota Trib. Renda do Capital & 0,1195 \\
\hline
\end{tabular}

: Fonte: Sistema de Contas Nacionais 2000-2005.

Table 3: Divisão das famílias por estado

\begin{tabular}{|l|c|c|c|c|c|c|c|}
\hline Estado & Popul. & Estado & Popul. & Estado & Popul. & Estado & Popul. \\
\hline AC & $0,34 \%$ & ES & $1,84 \%$ & PB & $1,99 \%$ & RO & $0,82 \%$ \\
\hline AL & $1,65 \%$ & GO & $2,99 \%$ & PR & $5,61 \%$ & RR & $0,20 \%$ \\
\hline AP & $0,30 \%$ & MA & $3,32 \%$ & PE & $4,62 \%$ & SC & $3,17 \%$ \\
\hline AM & $1,71 \%$ & MT & $1,50 \%$ & PI & $1,66 \%$ & SP & $21,87 \%$ \\
\hline BA & $7,61 \%$ & MS & $1,23 \%$ & RN & $1,63 \%$ & SE & $1,06 \%$ \\
\hline CE & $4,38 \%$ & MG & $10,50 \%$ & RS & $5,95 \%$ & TO & $0,69 \%$ \\
\hline DF & $1,23 \%$ & PA & $3,71 \%$ & RJ & $8,42 \%$ & Brasil & $100,00 \%$ \\
\hline
\end{tabular}

: Fonte: IBGE - Contas Regionais 2004.

\subsubsection{Horas Trabalhadas}

As horas trabalhadas para cada família foram determinadas utilizando os dados do Censo 2000 do IBGE (2002). O Censo, entretanto, não fornece diretamente o número de horas trabalhadas por Estado. Para resolver este problema, utilizou-se de dados do Censo que mostram horas trabalhadas por região e considerou-se que todos os Estados dentro de determinada região possui o mesmo número de horas trabalhadas. De posse destes dados pôde-se determinar as horas trabalhadas semanais por Estado. Para este modelo, preferiu-se trabalhar com a relação total de horas trabalhadas por total de horas semanais.

Table 4: Horas trabalhadas por região

\begin{tabular}{|l|c|c|c|c|c|}
\hline Região & Horas & Região & Horas & Região & Horas \\
\hline Norte & 0,2479 & Nordeste & 0,2399 & Sudeste & 0,2542 \\
\hline Sul & 0,2517 & Centro-Oeste & 0,256 & Brasil & 0,251 \\
\hline
\end{tabular}

: Fonte: Censo 2000.

\subsubsection{Produto e Arrecadação Estadual}

O produto per capita por Estado foi obtido das Contas Regionais 2004 (IBGE, 2006). Todos os valores calibrados neste modelo estão em proporção do PIB per capita nacional, que foi fixado em 1. Já os dados da arrecadação estadual per capita foram retirados de informações do Conselho Nacional de Política Fazendária (CONFAZ) para 2004 (CONFAZ, 2004). Tem-se, então, a tabela a seguir. 
Table 5: Produto e Arrecadação per capita por estado

\begin{tabular}{|c|c|c|c|c|c|c|c|c|}
\hline UF & Prod. & Arrec. & UF & Prod. & Arrec. & UF & Prod. & Arrec. \\
\hline AC & 0,5287 & 0,0396 & MA & 0,2825 & 0,0192 & RN & 0,552 & 0,0457 \\
\hline AL & 0,3985 & 0,0317 & MT & 1,0445 & 0,105 & RS & 1,3691 & 0,0873 \\
\hline AP & 0,6987 & 0,0327 & MS & 0,9194 & 0,1023 & RJ & 1,5046 & 0,0834 \\
\hline AM & 1,1752 & 0,0808 & MG & 0,9015 & 0,0676 & RO & 0,6412 & 0,0658 \\
\hline BA & 0,6527 & 0,0506 & PA & 0,5131 & 0,0341 & RR & 0,5017 & 0,0384 \\
\hline CE & 0,4286 & 0,0365 & PB & 0,4281 & 0,0312 & SC & 1,2497 & 0,0884 \\
\hline DF & 1,9602 & 0,1098 & PR & 1,1023 & 0,075 & SP & 1,4107 & 0,112 \\
\hline ES & 1,0575 & 0,1081 & PE & 0,589 & 0,0428 & SE & 0,6971 & 0,0438 \\
\hline GO & 0,771 & 0,0701 & PI & 0,2973 & 0,0248 & TO & 0,3881 & 0,0466 \\
\hline
\end{tabular}

: Fonte: Contas Regionais 2004.

\subsubsection{Taxa de Juros, Salário, Produtividade e Capital}

A taxa de juros real é dada pela diferença entre a taxa SELIC vigente em dezembro de 2004 e a inflação acumulada pelo IPCA em 2004, o que implica em $r=0,1170$. Por outro lado, dada a estrutura competitiva das firmas podemos calcular salários e estoque de capital por Estado como $w_{i}=\left(1-\theta \frac{y_{i}}{h_{i}}\right)$ e $k_{i}=\theta \frac{y_{i}}{r}$. A produtividade pode ser calculada da equação dos salários, 7. Substituindo valores construímos a próxima tabela.

Table 6: Salário, Produtividade e Estoque de Capital por Estado

\begin{tabular}{|c|c|c|c|c|c|c|c|c|c|c|c|}
\hline UF & Sal. & Prod. & Cap. & UF & Sal. & Prod. & Cap. & UF & Sal. & Prod. & Cap. \\
\hline AC & 1,119 & 0,599 & 2,146 & MA & 0,618 & 0,331 & 1,147 & RN & 1,208 & 0,647 & 2,241 \\
\hline AL & 0,872 & 0,467 & 1,618 & MT & 2,142 & 1,147 & 4,241 & RS & 2,855 & 1,53 & 5,559 \\
\hline AP & 1,479 & 0,792 & 2,837 & MS & 1,885 & 1,01 & 3,733 & RJ & 3,107 & 1,665 & 6,109 \\
\hline AM & 2,488 & 1,333 & 4,77 & MG & 1,862 & 0,997 & 3,66 & RO & 1,358 & 0,727 & 2,603 \\
\hline BA & 1,428 & 0,765 & 2,65 & PA & 1,086 & 0,582 & 2,083 & RR & 1,062 & 0,569 & 2,037 \\
\hline CE & 0,938 & 0,502 & 1,74 & PB & 0,937 & 0,502 & 1,738 & SC & 2,606 & 1,396 & 5,074 \\
\hline DF & 4,02 & 2,154 & 7,959 & PR & 2,299 & 1,231 & 4,476 & SP & 2,913 & 1,561 & 5,728 \\
\hline ES & 2,184 & 1,17 & 4,294 & PE & 1,289 & 0,69 & 2,391 & SE & 1,525 & 0,817 & 2,83 \\
\hline GO & 1,581 & 0,847 & 3,13 & PI & 0,651 & 0,348 & 1,207 & TO & 0,822 & 0,44 & 1,575 \\
\hline
\end{tabular}

: Fonte: Elaboração do autor.

\subsubsection{Depreciação e Taxa de Desconto Intertemporal}

A depreciação é obtida do investimento, $\delta=\frac{I}{k}=0,0439$, enquanto a taxa de desconto intertemporal sai da equação 12 no estado estacionário, $\beta=0,9441$. 


\subsubsection{Consumo, Fluxo comercial entre os Estados e Percentual do Consumo Tributado pelo ICMS}

O fluxo comercial entre os Estados foi obtido do trabalho "Balança Comercial Interestadual 1999" do CONFAZ (CONFAZ, 1999), que mostra quanto cada Estado vendeu para os demais. De posse destes dados foi montada a matriz constante do apêndice, que representa os coeficientes $a_{j i}$, ou seja, a proporção no consumo da família $i$ de bens produzidos no Estado $j$. Adotou-se a hipótese de que não houve modificação na distribuição percentual do consumo dos Estados por origem dos produtos entre 1999 e 2004.

Com relação ao consumo, nem todo ele sofre a incidência da tributação do ICMS, e mais ainda, as alíquotas não são todas fixas em $17 \%$. De fato, existe uma série de produtos que recebem alíquotas mais elevadas como comunicações, combustíveis e bebidas, e outras com alíquotas menores, especialmente alimentos e medicamentos. No modelo o parâmetro $s_{i}$ trata de fixar a alíquota efetiva que cada família paga em seu Estado, ao ser multiplicada por $17 \%$, a alíquota padrão do ICMS. Desta maneira $s_{i} 17 \%$ representa a alíquota efetiva do ICMS no Estado $i$.

Partindo dos dados de arrecadação estadual do CONFAZ (2004), com informações sobre a arrecadação do ICMS por Estado, e usando as equações 8 e 13 calculamos o consumo e a alíquota efetiva do ICMS por Estado, conforme tabela a seguir.

Table 7: Consumo e alíquota efetiva do ICMS

\begin{tabular}{|c|c|c|c|c|c|c|c|c|c|c|c|}
\hline UF & Cons. & Si & Alíq. & UF & Cons. & Si & Alíq. & UF & Cons. & Si & Alíq. \\
\hline AC & 0,33 & 0,73 & 0,124 & MA & 0,186 & 0,65 & 0,11 & RN & 0,358 & 0,78 & 0,132 \\
\hline AL & 0,256 & 0,84 & 0,143 & MT & 0,606 & 0,96 & 0,164 & RS & 0,863 & 0,5 & 0,086 \\
\hline AP & 0,455 & 0,42 & 0,071 & MS & 0,537 & 0,91 & 0,155 & RJ & 0,935 & 0,52 & 0,088 \\
\hline AM & 0,781 & 0,28 & 0,047 & MG & 0,551 & 0,64 & 0,109 & RO & 0,387 & 0,99 & 0,168 \\
\hline BA & 0,434 & 0,58 & 0,099 & PA & 0,325 & 0,62 & 0,105 & RR & 0,307 & 0,86 & 0,147 \\
\hline CE & 0,281 & 0,68 & 0,116 & PB & 0,284 & 0,59 & 0,1 & SC & 0,779 & 0,59 & 0,1 \\
\hline DF & 1,192 & 0,6 & 0,103 & PR & 0,691 & 0,55 & 0,093 & SP & 0,858 & 0,67 & 0,114 \\
\hline ES & 0,636 & 0,75 & 0,128 & PE & 0,391 & 0,6 & 0,102 & SE & 0,466 & 0,53 & 0,091 \\
\hline GO & 0,458 & 0,78 & 0,133 & PI & 0,189 & 0,91 & 0,155 & TO & 0,227 & 1,23 & 0,209 \\
\hline
\end{tabular}

: Fonte: Elaboração do autor.

São necessárias algumas observações a respeito destes números. Primeiro em relação ao Amazonas, local onde fica a Zona Franca de Manaus que goza de inúmeras isenções relacionadas ao ICMS, tanto nas vendas locais quanto para outros Estados, e explica a baixa alíquota efetiva obtida. Por outro lado, alguns Estados aparecem com alíquotas efetivas muito próximas ou superiores a 17\%, como Mato Grosso, Mato Grosso do Sul, Piaú, Rondônia e Tocantins. A explicação aqui reside tanto no fato de que nestes locais o consumo é relativamente pequeno em relação ao restante do país, seja por falta de renda ou por baixa população, quanto na própria característica do ICMS de que a arrecadação em um Estado decorre em boa medida do consumo nos outros Estados de mercadorias produzidas no Estado de origem. Ora, como a alíquota efetiva é calculada dividindo a arrecadação do ICMS de vendas internas e a outros Estados pelo consumo do próprio Estado, tem-se nestas duas explicações a razão para a alíquota elevada. Reforçando tal argumento, observa-se que ao dividir a arrecadação do ICMS fornecida pelo CONFAZ (CONFAZ, 2004) com os dados do produto por Estado das Contas Regionais 2004, tabela 5, Tocantins, Mato Grosso do Sul e Rondônia fornecem os valores mais elevados. 


\subsubsection{Tributação sobre o Consumo Estadual}

Como já visto, para o comércio interestadual valem as alíquotas definidas por resolução do Senado Federal e descritas na tabela 1 . Dentro dessa lógica, $\tau_{E t}^{j i}$ representa a parte da alíquota do ICMS do consumo da família $i$ que é repassada para o Estado produtor $j(12 \%$ ou $7 \%$ conforme a tabela 1$)$ e $\tau_{I t}^{j i}$ é a parte da alíquota do ICMS do consumo da família i de bens produzidos no Estado $j$ que fica com o Estado consumidor $i$ ( $5 \%$ ou $10 \%$ conforme a tabela 1, ou ainda, $17 \%$ se o bem for produzido e consumido dentro do próprio Estado). Para efeito do modelo, as alíquotas da tributação do ICMS devem ser multiplicadas pelo coeficiente $s_{i}$ para a representação das alíquotas efetivas.

\subsubsection{Peso do Consumo na Função Utilidade}

O peso do consumo na função utilidade, $\alpha$, foi obtido de forma a compatibilizar o consumo estadual calculado na tabela 7 com a participação do consumo no PIB dado pelo Novo Sistema de Contas Nacionais do IBGE para o ano de 2004 (IBGE, 2007), $c=0,6219$, tendo sido obtido o valor de $\alpha=0,3912$, valor em linha com os descritos na literatura.

\subsubsection{Participação dos Estados na Despesa Federal e Transferências Governamentais}

A participação dos Estados na despesa federal per capita com o bem único é dada pelo parâmetro $\gamma_{i}$, pode ser calculada usando a restrição agregada, equação 11. Já as transferências per capita do governo federal para as famílias em cada Estado, são obtidas pela restrição orçamentária das famílias equação 2 . Os valores estão dispostos na tabela 8.

Table 8: Participação dos estados na despesa federal e transferências governamentais

\begin{tabular}{|l|c|c|c|c|c|c|c|c|}
\hline UF & Desp. & Transf. & UF & Desp. & Transf. & UF & Desp. & Transf. \\
\hline AC & 1,2998 & 0,0783 & MA & 0,7348 & 0,0519 & RN & 1,207 & 0,1014 \\
\hline AL & 1,2182 & 0,0732 & MT & 2,4323 & 0,12 & RS & 1,104 & 0,181 \\
\hline AP & 1,2385 & 0,1035 & MS & 0,9756 & 0,1056 & RJ & 2,6832 & 0,1837 \\
\hline AM & 0,3175 & 0,1741 & MG & 0,6644 & 0,1101 & RO & 1,4678 & 0,095 \\
\hline BA & 0,6522 & 0,1199 & PA & 1,1842 & 0,076 & RR & 1,6688 & 0,0743 \\
\hline CE & 0,6262 & 0,0787 & PB & 0,6855 & 0,0786 & SC & 0,8833 & 0,1652 \\
\hline DF & 6,1727 & 0,2251 & PR & 0,9301 & 0,1457 & SP & 0,2756 & 0,1723 \\
\hline ES & 0,5543 & 0,1291 & PE & 1,1428 & 0,1082 & SE & 1,2562 & 0,1281 \\
\hline GO & 1,5184 & 0,0885 & PI & 0,9719 & 0,0546 & TO & 1,0109 & 0,0575 \\
\hline
\end{tabular}

: Fonte: IBGE - Contas Regionais 2004 e Elaboração do autor.

Em linha com o que seria de se esperar, Distrito Federal e Rio de Janeiro, capital e ex-capital brasileira, são os entes federativos mais beneficiados pela despesa e transferência federal.

\section{DISCUSSÃO DOS RESULTADOS}

Nas simulações que serão apresentadas, é importante destacar que a Zona Franca de Manaus foi preservada, o que para efeito de modelo, implica na manutenção do Estado do Amazonas na estrutura da tributação do ICMS atual.

A mudança do princípio da origem para o do destino não implicará em mudança na alíquota tributária efetiva do ICMS, que permanecerá com o mesmo valor de antes da mudança. O que ocorrerá será uma grande redistribuição da receita do ICMS entre os Estados. 
A nova distribuição das receitas terá um impacto significativo sobre as receitas estaduais, alterando, por conseguinte a despesa estadual. Entretanto, para cada Estado, a relação capital-trabalho não se alterará, embora para alguns Estados haja aumento da produção enquanto que para outros, redução. Para aqueles em que houve crescimento da produção, em razão principalmente do aumento da demanda do governo, teremos incremento do estoque de capital acompanhado por aumento das horas de trabalho. Dado que da maximização da utilidade das famílias, a relação entre a utilidade marginal do consumo e do lazer é dada pela relação entre o preço do consumo com tributos e o preço do lazer com tributos e que não houve alteração nestes preços, o aumento das horas de trabalho implica em redução do lazer e conseqüentemente em diminuição do consumo. Visto de outra forma, o aumento da produção leva a necessidade de mais investimentos que implica em redução do consumo. Assim, os Estados que aumentaram a sua arrecadação com a nova sistemática do ICMS, experimentarão também aumento da produção e redução do consumo. Para os Estados com perda de arrecadação, o processo é o inverso com redução da produção e aumento do consumo. A próxima Tabela mostra o efeito da mudança da cobrança do ICMS sobre o consumo e a produção no longo prazo.

Table 9: Variação percentual da produção e do consumo com o princípio do destino

\begin{tabular}{|l|c|c|c|c|c|c|c|c|}
\hline & Prod. & Cons. & UF & Prod. & Cons. & & Prod. & Cons. \\
\hline UF & $(\%)$ & $(\%)$ & & $(\%)$ & $(\%)$ & UF & $(\%)$ & $(\%)$ \\
\hline AC & 0,68 & $-0,22$ & MA & 0,65 & $-0,21$ & RN & 0,7 & $-0,22$ \\
\hline AL & 1,2 & $-0,38$ & MT & 0,6 & $-0,21$ & RS & $-0,31$ & 0,1 \\
\hline AP & 0,22 & $-0,07$ & MS & $-0,25$ & 0,09 & RJ & 0,21 & $-0,07$ \\
\hline AM & $-0,01$ & 0 & MG & $-0,06$ & 0,02 & RO & 0,91 & $-0,3$ \\
\hline BA & $-0,28$ & 0,09 & PA & 0,43 & $-0,14$ & RR & 0,78 & $-0,26$ \\
\hline CE & 0,14 & $-0,04$ & PB & 0,12 & $-0,04$ & SC & $-0,15$ & 0,05 \\
\hline DF & 0,7 & $-0,24$ & PR & $-0,21$ & 0,07 & SP & $-0,1$ & 0,03 \\
\hline ES & $-0,65$ & 0,22 & PE & 0,16 & $-0,05$ & SE & 0,23 & $-0,07$ \\
\hline GO & 0,08 & $-0,03$ & PI & 1,31 & $-0,41$ & TO & 1,34 & $-0,44$ \\
\hline
\end{tabular}

: Fonte: Elaboração do autor.

As figuras a seguir mostram a evolução do produto e consumo para alguns Estados selecionados.

Os resultados mostram que a mudança na sistemática da tributação do ICMS praticamente não afeta o produto e o consumo dos Estados. Assim, apesar de ser uma alteração tributária de grande monta, seu efeito direto sobre os agentes é reduzido. Não será diretamente que o princípio do destino afetará as desigualdades regionais. Na verdade, conforme se vê na Tabela abaixo, a sua força está na alteração da distribuição das receitas do ICMS entre os entes federativos.

Verifica-se que os Estados do Sul, do Sudeste, exceto o Rio de Janeiro, e mais Bahia e Mato Grosso do Sul perderiam com a implantação do princípio do destino. Com a exceção da Bahia, nenhum dos perdedores se situa entre os mais pobres do Brasil. Já os maiores ganhadores são Acre, Alagoas, Maranhão, Distrito Federal, Piauí e Roraima. Exceto pela presença do Distrito Federal, os demais Estados pertencem ao grupo mais pobre da federação. Assim, a adoção do princípio do destino levaria a uma distribuição mais eqüitativa dos recursos tributários entre os entes federativos. Quanto ao Distrito Federal, tratase de uma região que praticamente não possui indústrias e importa quase tudo o que consome, como qualquer Estado pobre. Entretanto, a capital federal possui a mais alta renda per capita do Brasil.

Observa-se, assim, que o grande potencial do princípio do destino para a redução das desigualdades regionais não está nos seus efeitos diretos sobre o produto ou o consumo dos Estados mais pobres, e sim no seu efeito indireto, ao carrear grandes somas de recursos para estes Estados, que passam a dispor 
Figure 1: Evolução do produto - estados selecionados
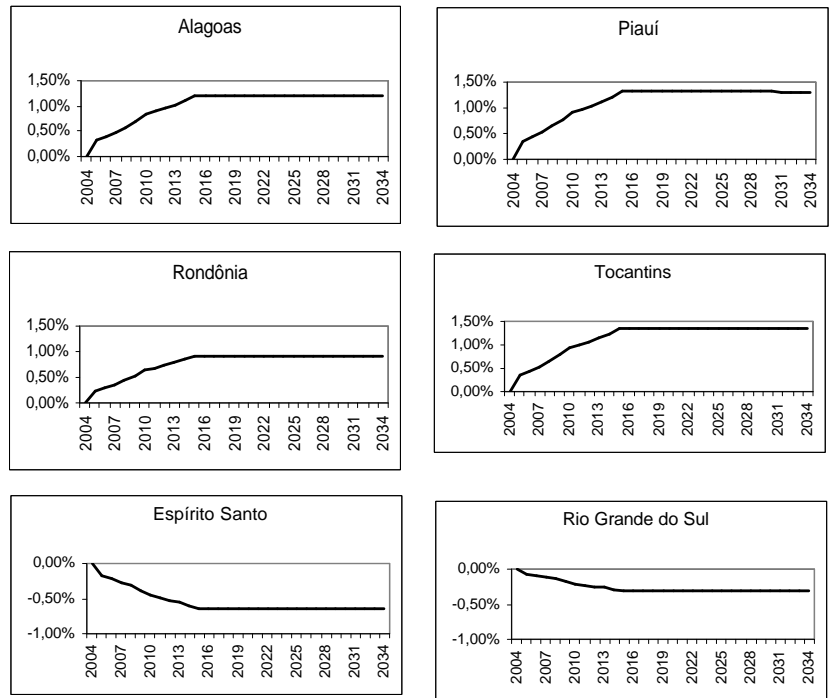

Figure 2: Evolução do consumo - estados selecionados
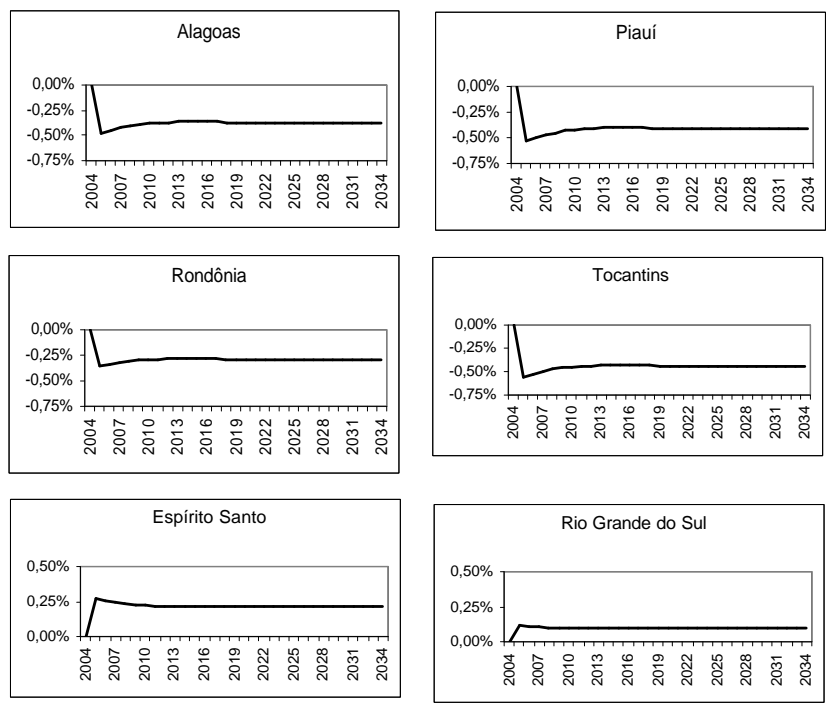

de quantias próprias fundamentais para o seu desenvolvimento. Há muito que se fazer em áreas como infra-estrutura, educação, saúde, saneamento e outras, e a mudança no ICMS pode gerar boa parte dos recursos necessários.

Entretanto, algumas ressalvas são necessárias. É preciso muita parcimônia dos Estados beneficiados com o uso destes recursos, evitando o desperdício e a corrupção. O mesmo se aplica com os prejudicados pela alteração, que deverão se reestruturar para conviver com menos recursos, tendo a União papel 
Table 10: Variação na arrecadação estadual - R\$ milhões

\begin{tabular}{|l|c|c|c|c|c|c|c|}
\hline & Antes 2004 & Variação & Variação & & Antes 2004 & Variação & Variação \\
\hline UF & & $\%$ & RS milhões & & UF & $\%$ & RS milhões \\
\hline AC & 257 & $15,90 \%$ & 41 & PB & 1.145 & $0,70 \%$ & 8 \\
\hline AL & 973 & $32,80 \%$ & 319 & PR & 7.824 & $-5,50 \%$ & -433 \\
\hline AP & 184 & $6,50 \%$ & 12 & PE & 3.667 & $2,30 \%$ & 83 \\
\hline AM & 2.613 & $0,00 \%$ & 1 & PI & 762 & $36,80 \%$ & 280 \\
\hline BA & 7.133 & $-5,70 \%$ & -410 & RN & 1.395 & $17,70 \%$ & 247 \\
\hline CE & 2.994 & $0,40 \%$ & 12 & RS & 9.638 & $-7,60 \%$ & -732 \\
\hline DF & 2.581 & $22,30 \%$ & 576 & RJ & 13.052 & $7,60 \%$ & 992 \\
\hline ES & 3.732 & $-13,40 \%$ & -499 & RO & 1.058 & $17,60 \%$ & 186 \\
\hline GO & 3.978 & $0,00 \%$ & 1 & RR & 151 & $24,00 \%$ & 36 \\
\hline MA & 1.192 & $18,60 \%$ & 222 & SC & 5.258 & $-2,70 \%$ & -142 \\
\hline MT & 2.973 & $12,20 \%$ & 363 & SP & 45.922 & $-3,00 \%$ & -1.398 \\
\hline MS & 2.349 & $-4,00 \%$ & -94 & SE & 873 & $5,90 \%$ & 51 \\
\hline MG & 13.222 & $-0,60 \%$ & -83 & TO & 606 & $27,00 \%$ & 164 \\
\hline PA & 2.406 & $10,60 \%$ & 256 & BR & 137.938 & $0,10 \%$ & 59 \\
\hline
\end{tabular}

: Fonte: Elaboração do autor.

preponderante no auxílio durante a transição. Por último, a implementação do princípio do destino gera um potencial de arrecadação extra para os Estados ganhadores, que só se tornará efetiva com a modernização da Administração Tributária estadual e uma legislação que evite a evasão fiscal nas transações interestaduais. Uma das alternativas que já foram propostas é o mecanismo conhecido como "modelo do barquinho", proposto por Varsano (1999), sob a hipótese de implementação de um IVA - Dual, ou seja, um IVA no destino com parte da alíquota federal e parte estadual.

\section{CONSIDERAÇÕES FINAIS}

O principal imposto brasileiro, o ICMS, está assentado atualmente em parte sobre a produção e em parte sobre o consumo, em razão da adoção em parte do princípio da origem na distribuição das receitas arrecadadas por este imposto nas transações interestaduais.

Neste artigo foram realizadas simulações alterando a sistemática de distribuição de receitas para a adoção plena do princípio do destino, no qual toda a arrecadação do ICMS seria destinada ao Estado consumidor das mercadorias.

Entretanto, tal alteração não é neutra do ponto de vista dos Estados. Como se viu nos resultados, os Estados produtores tem perdas de arrecadação que chegam a quase R\$ 1,4 bilhão, como no caso de São Paulo, em valor absoluto, ou 13,4\%, para o Espírito Santo, em termos relativos. Já os ganhadores são os Estados consumidores, em geral, os menos desenvolvidos da federação, mas que também inclui alguns dos mais desenvolvidos e com relativamente pouca produção de mercadorias. Em termos absolutos, os maiores ganhos estão no Rio de Janeiro, quase R\$ 1 bilhão, e Distrito Federal, R\$ 576 milhões. Em termos relativos, os mais favorecidos são Piauí, com um acréscimo de $37 \%$ na sua arrecadação, e Alagoas com um incremento de $33 \%$.

Dado o impacto significativo nas finanças dos Estados prejudicados pelo princípio do destino, é recomendável que a transição de um regime para outro seja feita num intervalo de tempo razoável e 
com o apoio do governo federal, de forma a minimizar os custos de bem-estar que serão impostos à população. Políticas de aumento da eficiência na Administração Tributária são importantes tanto para ganhadores quanto para perdedores. Os primeiros para que a receita potencial com a nova sistemática se torne real, e os últimos como tentativa de recuperar parte das perdas.

\section{BIBLIOGRAPHY}

CONFAZ (1999). Balança Comercial Interestadual 1999.

CONFAZ (2004). Boletim do ICMS 2004.

IBGE (2002). Censo Demográfico 2000.

IBGE (2006). Contas Regionais 2004.

IBGE (2007). Sistema de Contas Nacionais 2000-2005.

Miranda, M. J. \& Fackler, P. L. (2002). Applied Computational Economics and Finance. MIT Press.

Paes, N. \& Siqueira, M. L. (2005). Análise dos efeitos econômicos da implantação do princípio do destino na cobrança do icms e suas implicações sobre a pobreza e a desigualdade de renda. Economia, 6:129144.

Salvi, A. (2005). Mecanismos de transferências de renda inter-regionais: Experiências brasileiras e européias. Escola de Administração Fazendária - ESAF, 2005.

Varsano, R. (1997). A guerra fiscal do ICMS: Quem ganha e quem perde. IPEA - Texto para Discussão 500.

Varsano, R. (1999). Subnational taxation and treatment of interstate trade in Brazil: Problems and a proposed solution. The World Bank Conference - Valdívia, Chile.

\section{A. TABELAS}




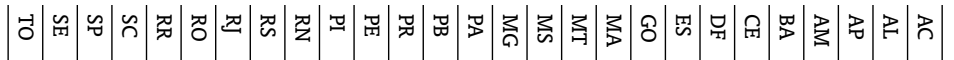

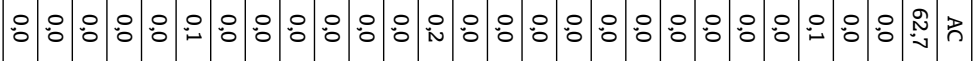

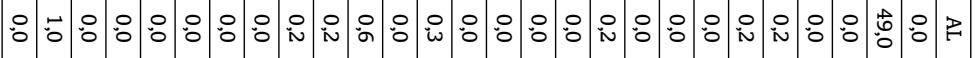

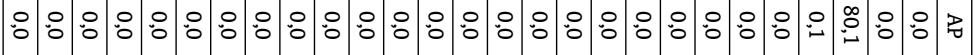

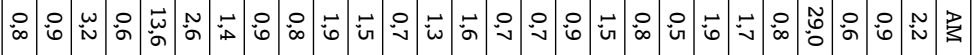

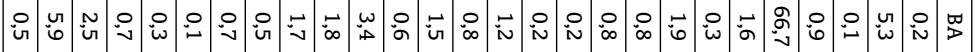

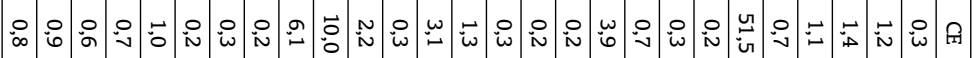

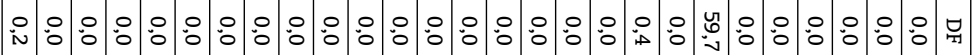

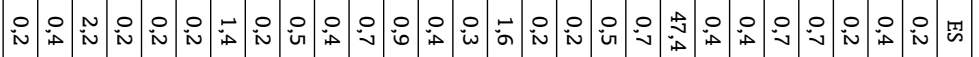

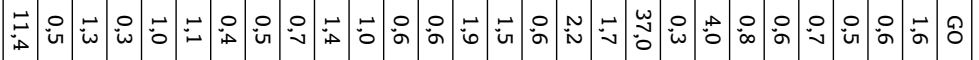

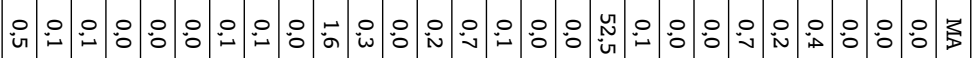

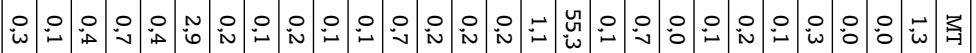

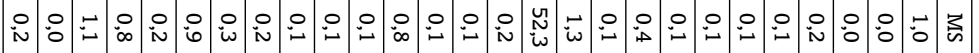

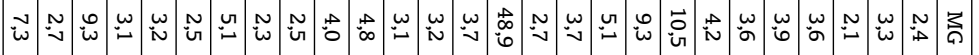

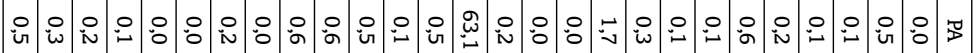

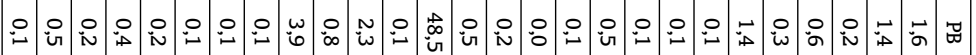

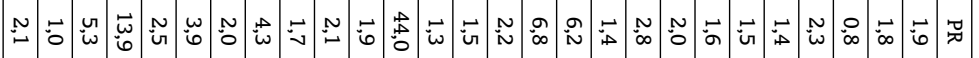

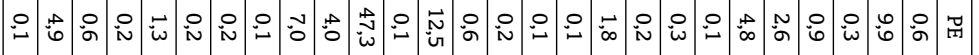

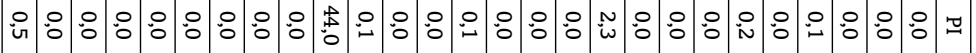

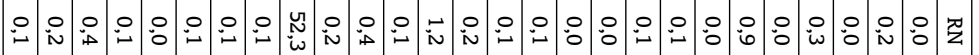

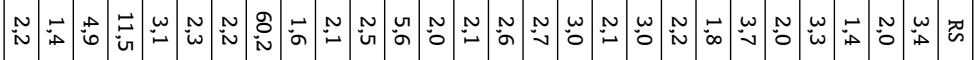

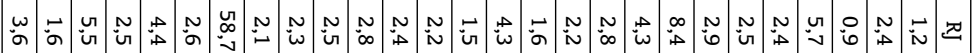

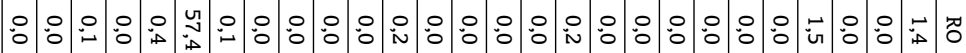

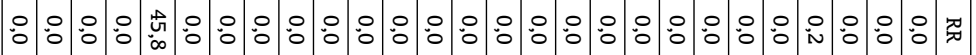

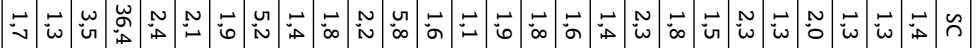

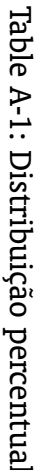

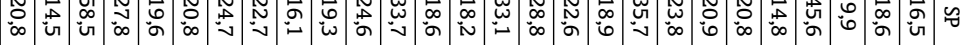

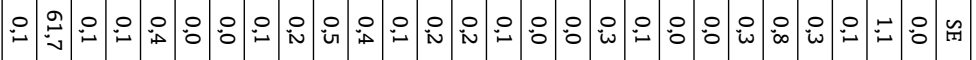

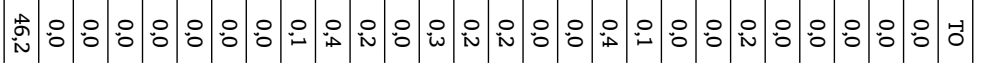

